Open Access

\title{
Surveying happiness in China: comparing measures of subjective well-being
}

\author{
Becky Hsu', Weiwei Zhang ${ }^{2^{*}}$ and Christine Kim
}

\author{
* Correspondence: \\ wzhang@stlawu.edu \\ ${ }^{2}$ Sociology Department, St. \\ Lawrence University, 23 Romoda \\ Drive, Canton, NY 13617, USA \\ Full list of author information is \\ available at the end of the article
}

\begin{abstract}
The objectives of this study are to investigate (1) whether subjective well-being (SWB) measures in surveys on China are interchangeable and (2) whether these measures work together as a construct. To accomplish these tasks, we examine SWB measures 1990-2016 for basic statistics, correlations between pairs of happiness terms within the same surveys, and test the internal consistency of five variables in the Blessed Happiness survey. Findings indicate that while the terms are not interchangeable, they work together to create a reasonably reliable construct.
\end{abstract}

\section{Background}

Influential cross-national studies have combined measures for subjective well-being (SWB) or used them interchangeably (Deaton 2008; Hagerty and Veenhoven 2003). A prominent example is Richard Easterlin's work, which uses cross-national data to argue for the "happiness-income paradox," or "Easterlin paradox." Easterlin has found that countries with higher gross domestic product are not happier, even though wealthier individuals are happier within each country, with China as the most significant case (Easterlin 1974; Easterlin et al. 2010). Because of the scarcity of data over time, these studies have combined data sets using different terms for happiness, but treat them as the same concept. Easterlin et al. (2012) combines seven data sets using three terms: satisfaction (manyi) (World Values Survey), happiness (xingfu) (Asiabarometer), and the "ladder of life" (Pew). The most recent update finds that there is a U-shaped pattern in China, falling in the 2000-05 period and then moving upward again since. As part of the analysis, the authors present trend lines from 1990 to 2015 using five data sets; one trend line begins with Gallup1 (satisfaction manyi) and continues with CGSS (happiness xingfu) (Easterlin et al. 2017: 50).

The different SWB terms have qualitatively different meanings and histories, so we wonder whether they can be used interchangeably. Additionally, some SWB terms connote emotion or mood, while others are about a broad evaluation of life. Finally, we wonder whether the different measures can be used as part of a construct for a larger assessment of well-being on the part of the respondent.

\section{Comparisons between subjective well-being measures}

There are several data sets in China that include more than one SWB measure, which makes it possible to compare measures. In this paper, we examine five SWB

(c) The Author(s). 2017 Open Access This article is distributed under the terms of the Creative Commons Attribution 4.0 International License (http://creativecommons.org/licenses/by/4.0/), which permits unrestricted use, distribution, and reproduction in any medium provided you give appropriate credit to the original author(s) and the source, provide a link to the Creative Commons license, and indicate if changes were made. 
terms-happiness (xingfu), satisfaction (manyi), good life (hao sheng huo), happy (yukuai), enjoying life (xiangshou shenghuo) - which all have slightly different connotations. Because these measures have not been compared before, we take a "first look" and compare all the terms to each other. Each term is different from the others, and some, like happiness (xingfu) have especially complex histories.

Happiness (xingfu) is a modern word from Japan's late imperial period that began to be used in the early twentieth century (Chen 2014). A concept influenced by Confucianism, Daoism, and Buddhism, xingfu encompasses friendship, learning, self-knowledge, virtue and living in simplicity. Colloquially, to say that someone is happy (xingfu) could mean that one feels very good about one's life, that their relationships with friends and family (especially parents) are good. To say, "my family is happy (wo jiating hen xingfu)," might indicate that my parents are healthy, my children are well, that I have lots of friends, and personal accomplishments (like career or material wealth).

Happiness (xingfu) is a term that has been used in the past decade quite a bit by the government, which may affect the way people respond to it. Since around 2000, the Chinese government has been consciously promoting a notion of happiness as something given to the people by the state (Sun 2014). The official discourse adopts a Confucian language of benevolent politics (仁政ren zheng), which suggests that the people receive a happiness made possible by the state, emphasizing the common good and social stability. On March 17, 2013, China's new leader, Xi Jinping, pronounced a new slogan of "the China Dream" at the Party Congress with this statement: "The China Dream is about strengthening the state and making it powerful and prosperous; achieving a renaissance of the Chinese nation; and promoting the happiness (xingfu) of the people" (Xi 2013: 71). This use of the term happiness (xingfu) is part of rhetoric justifying the government's ruling power, using it as a characteristic of a paternal relationship. Xingfu has therefore been part of the endeavors to instruct the people about how to think about happiness and the common good.

The other broad life evaluations are not quite as complex as happiness (xingfu), but they do have a variety of connotations. Life satisfaction (manyi) is translated as "satisfaction" in the Xinhua dictionary. As with its English equivalent, manyi denotes met expectations and hopes. Good life (haoshenghuo) is a broad, overall evaluation of life that refers to material or physical comfort, including good health and material or financial security.

Three terms we examine primarily denote positive emotion or good mood: happy (kuaile) and happy (yukuai). Enjoying life (xiangshou shenghuo) is also a feeling, but it is more specific in that it connotes being anxiety-free and comfortable. It is the picture of someone luxuriating in the sun on a beach and saying to herself, "This is the life."

\section{The role of emotion in self-evaluation}

People use different criteria for self-evaluation, so "cultural prototypes" for life satisfaction vary around the world (Suh 2000: 66). The evaluation of life can vary in regards to how important emotion is in that assessment. In English-language surveys, reliability tests between affect and evaluation show that both types of measures show a serial correlation of about 0.60 (not as high as reliability ratios found for education and income, but high enough to support the research on SWB that combines or substitutes terms 
(Krueger and Schkade 2008). However, tests of SWB measures have not been done with Chinese-language surveys.

Evidence from cross-cultural psychology suggests that self-judgment happens differently in China and locations where the English-language SWB surveys are used. Some SWB measures focus on affect (mood or emotional state) while others measure a broad evaluation of one's life. In China, relational and interdependent aspects of the self are emphasized rather than the unique, individuated aspects of the self (Suh et al. 1998). Emotion is not as important to self-judgment and interpersonal considerations ("How am I viewed by others?") in China, and external standards are salient concerns. Relationship harmony predicts life satisfaction more in Hong Kong than in the United States (Kwan et al. 1997). In contrast, in North America, individuals treat their emotions as important evidence for evaluating well-being (Suh 2000). If emotion is not as important to self-judgment in China, the SWB terms referring to emotion, like happy (kuaile), happy (yukuai), or enjoying life (xiangshou shenghuo) may not be as similar to self-evaluation terms like happiness (xingfu), life satisfaction (manyi), or good life (hao shenghuo).

\section{Working together as a construct}

If the SWB measures are not interchangeable, they could work together as a construct. Positive psychology has conceptualized human flourishing as a construct that is made up of five components: positive emotions, feeling engaged with one's activities, social relationships going well, meaning, a sense of accomplishment, and physical well-being (health and wealth) (Seligman 2011).

In China, people may not use emotion as criteria for self-evaluation as much as in North America, but people may still consider emotion one component among others that are about a broader well-being. As for other elements that approximate the construct people may be using to evaluate their lives, we may be able to tap into feeling engaged with one's activities and a sense of accomplishment with a measure on career satisfaction. Finally, though few cross-national surveys include a question on meaning $(y i y i)$, this sense of whether one feels that one's life has a sense of significance, is part of something larger than oneself, and is important would be good to include where possible.

\section{Data and Methods}

We list basic statistics from eight data sets where SWB variables are readily available (Table 1).

For comparisons between terms, we utilize three data sets with national sampling that include more than one SWB variable in the same year: World Values Survey, Asiabarometer, and Blessed Happiness.

The cross-national and longitudinal World Values Survey (WVS) includes China and SWB variables beginning in 1990 (though its origins are in the European Values Survey, which began in 1981). We optimize on 1995, 2007, and 2012, the years where more than one SWB term was used. In 1995, the survey includes questions on happiness (xingfu) and life satisfaction (manyi). In 2007 and 2012, both happy (yukuai) and satisfaction (manyi) are present (Table 2). 


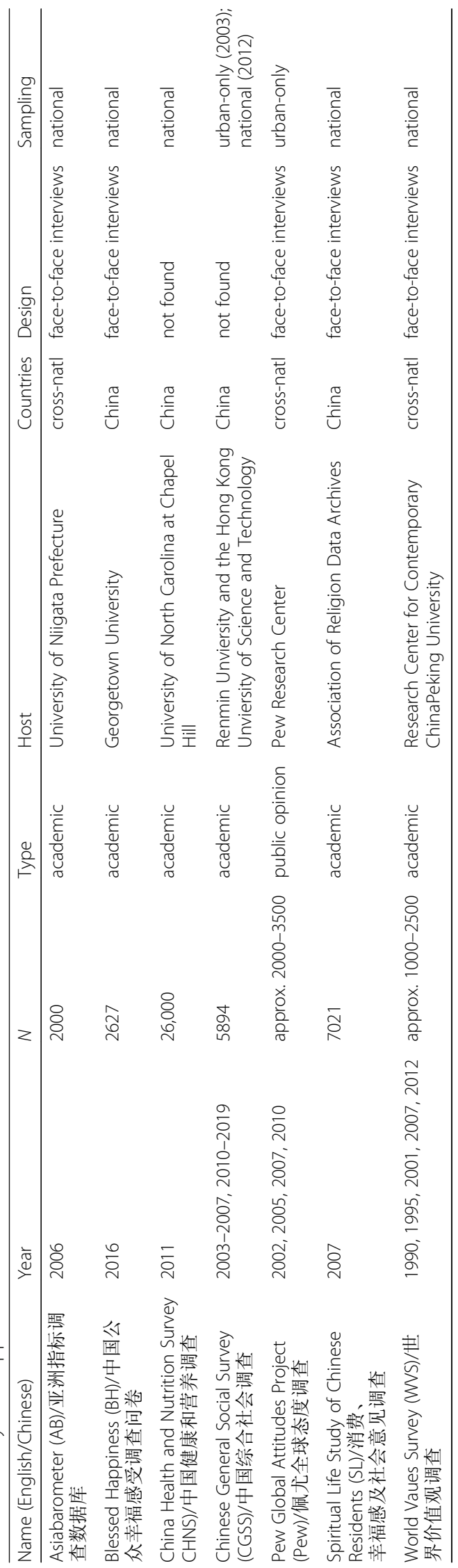




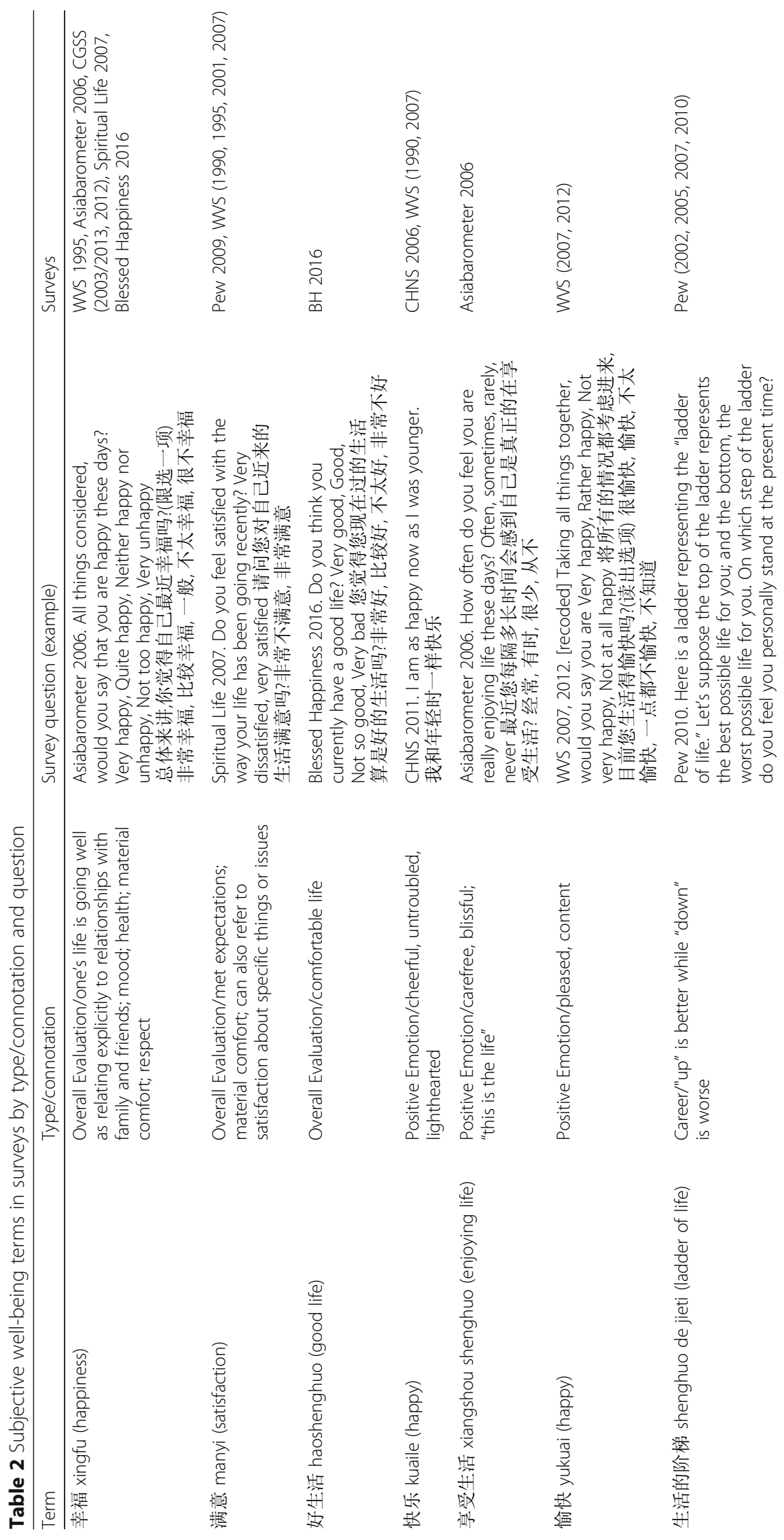




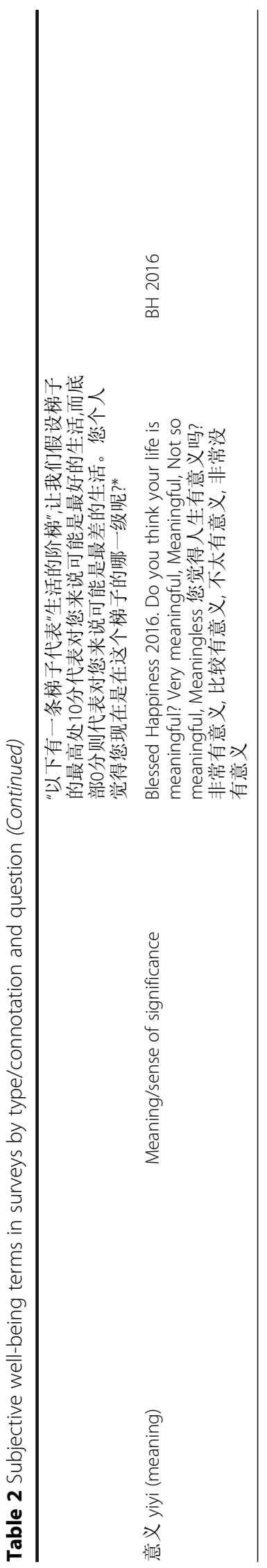


We note two issues that SWB researchers will need to be aware of when using the WVS China data. First, while there may seem to be two SWB terms in the WVS 1990, there is no Chinese questionnaire available to the public. It is difficult to tell which terms are being used from the English translation in the "results" file that is available on the website, so we do not use it. Second, the English translation in the 1995 codebook for V11 may be misleading for SWB researchers because, while it seems like a general SWB question, it is actually a specific question about the respondent's health. The results file translates the question as "Taking all things together, would you say that you are: Very good, Good, Fair, Poor, Very poor," but examining the Chinese, the question is “整体上看,您最近的健康状况如何?,” which is more accurately, “Lately, would you say that your health has been: Very good, Good, Average, Not good, Very Bad."

Asiabarometer (AB) includes two SWB measures in its survey on China, which is included in its comparative survey covering East, Southeast, South, and Central Asia every year between 2003 and 2008 (but not every country is included each year). The survey covers topics such as identity, social actions, customs and rules, life values, economic conditions, social infrastructure, and political consciousness. In its nationally representative 2006 survey of China, AB includes one overall evaluation variable (happiness xingfu) and one measuring positive emotion (enjoying life xiangshou shenghuo).

A nationally representative 2016 survey, Blessed Happiness (BH), is an offshoot of a three-year ethnographic project on how people in China define happiness and the good life lasting from 2013 to 2016. The survey includes two measures for overall evaluation (happiness xingfu and good life haoshenghuo), one measure for positive emotion (happy kuaile), one measure for satisfaction (manyi) with one's job, and, perhaps most unique among the surveys, a measure for the respondent's sense of meaning, "Do you feel your life has meaning? Has a lot of meaning, Has more meaning, Doesn't have much meaning, Very much lacking meaning." The survey includes a variety of social interaction measures. The data will be publicly available in 2019 .

\section{Results}

We provide basic statistics for the SWB terms in Table 3. The corresponding scatterplot (Fig. 1) shows the three SWB measures over time in all data sets: happiness (xingfu), satisfaction (manyi), and ladder, from 1990 to 2016. We examine the highest SWB category because it is the most "comparable" of the responses (the "happiest" response available to respondents). The middle two responses are harder to compare (for example, "neither" is not the same as "quite happy" or "somewhat satisfied"). Additionally, if there are cultural traditions of emotional moderation, it may be that those who do select the highest level of SWB might feel strongly about it. We observe that, in each general time period, respondents tend to feel most satisfied (manyi) relative to the highest responses for happiness (xingfu) and ladder questions. This begins to suggest that satisfaction (manyi) seems to be conceptually different from the other concepts.

\section{Moderate-to-low correlations between terms}

Simple correlations (Pearson's R) between pairs of happiness terms in the same data set and year are in Table 4. The correlation between terms is not high. That the correlations between SWB terms are at best moderate suggest that people do not respond the 
Table 3 Basic statistics

\begin{tabular}{|c|c|c|c|c|}
\hline $\begin{array}{l}\text { National sample } \\
\text { Happiness xingfu }\end{array}$ & Very/Quite happy & Neither & Not happy & Very unhappy \\
\hline WV 1995 & 23 & 61 & 14 & 2 \\
\hline AB 2006 & 19 & 40 & 34 & 7 \\
\hline SL 2007 & 33 & 47 & 16 & 4 \\
\hline CGSS 2012 & 16 & 58 & 17 & 10 \\
\hline BH 2016 & 16 & 69 & 13 & 2 \\
\hline Happy yukuai & Very happy & Rather happy & Not too happy & Not at all happy \\
\hline WV 2007 & 21 & 55 & 19 & 4 \\
\hline WVS 2012 & 16 & 69 & 13 & 1 \\
\hline Satisfied manyi & Very satisfied & Somewhat satisfied & Somewhat dissatisfied & Very dissatisfied \\
\hline WVS 1990 & 30 & 52 & 15 & 3 \\
\hline WV 1995 & 28 & 45 & 21 & 7 \\
\hline WV 2001 & 23 & 45 & 24 & 8 \\
\hline WVS 2007 & 24 & 50 & 19 & 8 \\
\hline WVS 2012 & 18 & 57 & 21 & 3 \\
\hline Happy kuaile & $\begin{array}{l}\text { Strongly agree/ } \\
\text { Agree }\end{array}$ & Neutral & Disagree & Strongly disagree \\
\hline CHNS 2010 & 39 & 29 & 30 & 2 \\
\hline BH 2016 & 18 & 72 & 10 & 0 \\
\hline Good life haoshenghuo & Very good & Good & Not so good & Very bad \\
\hline BH 2016 & 16 & 66 & 17 & 1 \\
\hline $\begin{array}{l}\text { Enjoying life xiangshou } \\
\text { shenghuo }\end{array}$ & Often & Sometimes & Rarely & Never \\
\hline AB 2006 & 23 & 52 & 22 & 4 \\
\hline Meaning yiyi & Very meaningful & Meaningful & Not so meaningful & Meaningless \\
\hline BH 2016 & 24 & 68 & 7 & 0 \\
\hline \multicolumn{5}{|l|}{ Urban-only sample } \\
\hline $\begin{array}{l}\text { Ladder of life shenghuo } \\
\text { de jieti }\end{array}$ & highest 10,9, 8 & $7,6,5$ & 4,3 & lowest 2, 1, 0 \\
\hline Pew 2002 & 13 & 62 & 16 & 9 \\
\hline Pew 2005 & 14 & 61 & 17 & 8 \\
\hline Pew 2007 & 17 & 66 & 13 & 4 \\
\hline Pew 2010 & 13 & 73 & 14 & 1 \\
\hline
\end{tabular}

This table gives the percentage of responses to happiness questions by data source

same to the various SWB concepts. In the WVS 1995, happiness (xingfu) and satisfaction (manyi) are correlated at 0.44. In the BH 2016, the good life (hao shenghuo) is correlated with happiness (xingfu) at 0.42 . We note, however, that Pearson's $\mathrm{R}$ assumes a linear relationship, so we are not sure how this statistic fits with how conceptually related two measures of happiness are.

\section{Emotion and evaluation}

The findings offer some support for the idea that in China, emotions do not make up a large portion of self-evaluation. The results indicate that the evaluation terms of happiness (xingfu), satisfaction (manyi), and good life (hao shenghuo) are not really 


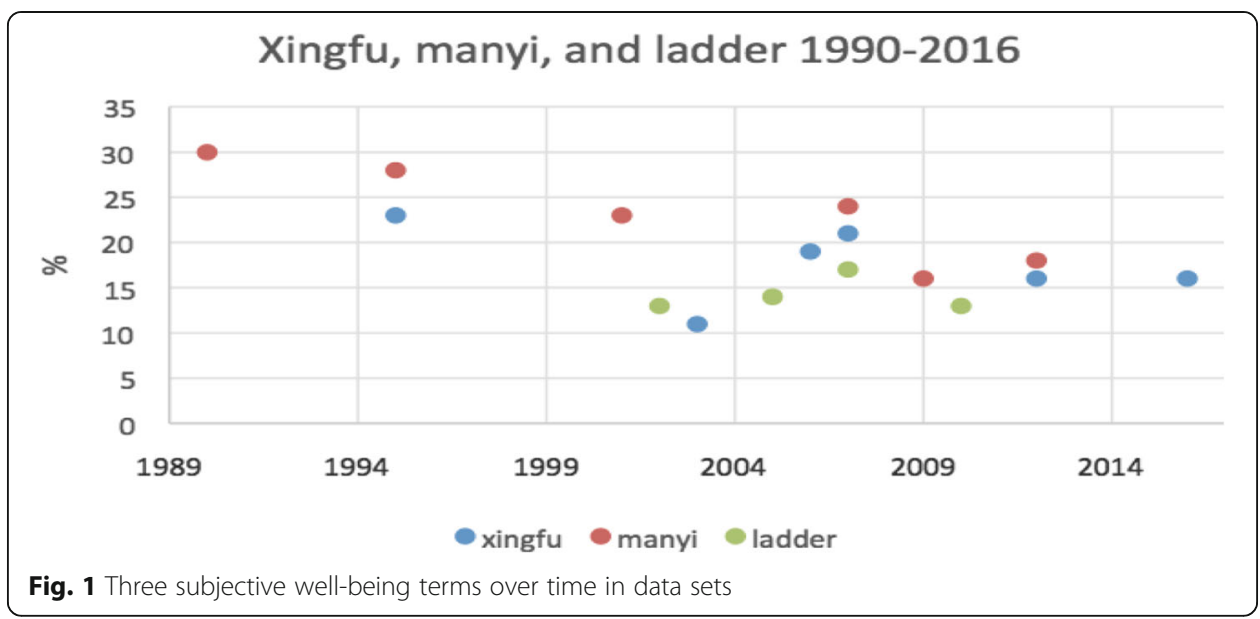

equivalent, but that they are even less similar to the emotion terms enjoying life (xiangshou shenghuo) and happy (kuaile).

In the WVS, the affective happy (yukuai) is correlated with satisfaction (manyi) at 0.53 in 2007, and 0.50 in 2012. In the Asiabarometer 2006, happiness (xingfu) and enjoying life (xiangshou shenghuo) are correlated at 0.45. In the BH 2016, happiness (xingfu) is correlated with good life (hao shenghuo) at 0.42 , meaning (yiyi) at 0.36 , and satisfied (manyi) with job at 0.35 , and least of all with happy (kuaile) at 0.33 . It is not much of a difference, but the least correlated variable with the evaluative happiness (xingfu) is indeed happy (kuaile), which gives some support to the argument that the evaluative and the affective are not the same thing in China. People who respond that they have a very good life do not necessarily say they feel positive emotions.

\section{Coefficients of reliability}

The five SWB variables in BH 2016 allowed us to further examine the "relatedness" of these measures. We generated Cronbach's alpha, a measure of reliability or internal consistency, for happiness (xingfu), meaning (yiyi), happy (kuaile), satisfied (manyi) with job, and good life (hao shenghuo). With all five variables, the Cronbach's alpha coefficient is 0.73 , indicating a reasonably reliable SWB concept. Removing just one variable lowers the alpha to between 0.65 to 0.72 (for the latter alpha, the combination of four variables are happiness, meaning, satisfied with job, and good life-that is, including happy seems to reduce internal consistency). Removing two variables reduced the alphas even more. The five-variable alpha may be higher simply because we increased the number of items in the analysis. However, combined with the moderate-to-low correlation numbers in Table 4, this means that the high alpha coefficients are not solely because the variables are highly correlated to begin with. We believe that the face validity is reasonably high, since these measures may tap into different elements of how people assess a flourishing life.

\section{Modal response: second highest}

We observe that the modal response for happiness (xingfu), satisfaction (manyi), and ladder is the second highest response (Fig. 2). In other words, "neither" happy nor 
Table 4 Correlations and descriptive statistics

\begin{tabular}{|c|c|c|c|c|c|}
\hline WVS 1995 & 1 & 2 & & & \\
\hline \multicolumn{6}{|l|}{ 1. happiness (xingfu) } \\
\hline 2. satisfaction (manyi) & $0.44^{a}$ & & & & \\
\hline Mean (SD) & $1.95(0.66)$ & $6.83(2.42)$ & & & \\
\hline Range & $1-4$ & $1-10$ & & & \\
\hline N & 1491 & 1495 & & & \\
\hline WVS 2007 & 1 & 2 & & & \\
\hline \multicolumn{6}{|l|}{ 1. happy (yukuai) } \\
\hline 2. satisfaction (manyi) & $0.53^{\mathrm{a}}$ & & & & \\
\hline Mean (SD) & $2.06(0.75)$ & $6.76(2.40)$ & & & \\
\hline Range & $1-4$ & $1-10$ & & & \\
\hline $\mathrm{N}$ & 1978 & 1937 & & & \\
\hline WVS 2012 & 1 & 2 & & & \\
\hline \multicolumn{6}{|l|}{ 1. happy (yukuai) } \\
\hline 2. satisfaction (manyi) & $0.50^{\mathrm{a}}$ & & & & \\
\hline Mean (SD) & $1.99(0.58)$ & $6.85(1.98)$ & & & \\
\hline Range & $1-4$ & $1-10$ & & & \\
\hline $\mathrm{N}$ & 2272 & 2252 & & & \\
\hline$A B 2006$ & 1 & 2 & & & \\
\hline \multicolumn{6}{|c|}{ 1. enjoying life (xiangshou shenghuo) } \\
\hline 2. happiness (xingfu) & $0.45^{\mathrm{a}}$ & & & & \\
\hline Mean (SD) & $2.07(0.77)$ & $1.50(0.67)$ & & & \\
\hline Range & $1-4$ & $1-4$ & & & \\
\hline $\mathrm{N}$ & 1997 & 1998 & & & \\
\hline BH 2016 & 1 & 2 & 3 & 4 & 5 \\
\hline \multicolumn{6}{|l|}{ 1. happy (kuaile) } \\
\hline 2. happiness (xingfu) & $0.33^{\mathrm{a}}$ & & & & \\
\hline 3. satisfied (manyi) with job & $0.27^{\mathrm{a}}$ & $0.35^{\mathrm{a}}$ & & & \\
\hline 4. meaning (yiyi) & $0.24^{a}$ & $0.36^{\mathrm{a}}$ & $0.33^{\mathrm{a}}$ & & \\
\hline 5. good life (hao shenghuo) & $0.28^{* * *}$ & $0.42^{\mathrm{a}}$ & $0.44^{\mathrm{a}}$ & $0.44^{\mathrm{a}}$ & \\
\hline Mean (SD) & $3.07(0.53)$ & $3.98(0.63)$ & $2.84(0.61)$ & $3.17(0.55)$ & $2.98(0.60)$ \\
\hline Range & $1-4$ & $1-5$ & $1-4$ & $1-4$ & $1-4$ \\
\hline N & 2597 & 2635 & 2189 & 2557 & 2561 \\
\hline
\end{tabular}

${ }^{a}$ This table presents the correlations between pairs of measures examined in this paper that exist in the same data set

unhappy (xingfu), "somewhat satisfied" (manyi), and "7,6,5" for ladder). While we do not have a definite explanation for this, one factor that may contribute to this is that people are less likely to choose the highest response because of the traditional cultural preference to be in moderate, reflecting the idea that happiness and unhappiness follow upon one another, so events in one's life should not be interpreted as either extremely good or bad.

In Chinese tradition, there is the idea that happiness and unhappiness follow upon one another, so events should be interpreted as neither extremely good nor extremely bad, and should not be received with excessive emotion. Fortune can turn quickly into misfortune because of uncontrollable external factors, and vice versa, as the 


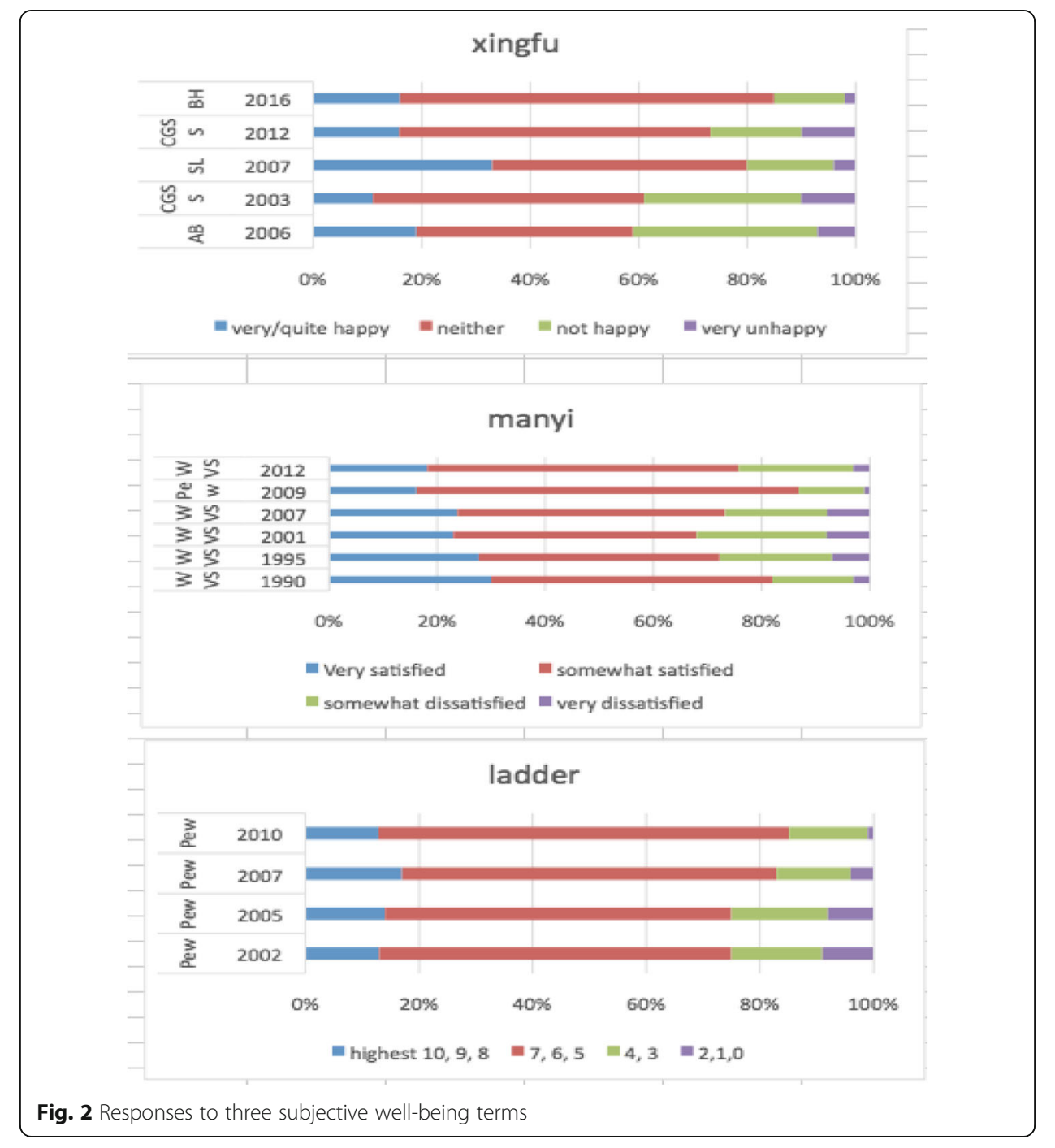

well-known story of old man Saiweng shows: when his horse ran away, his neighbors were sad for him, but he thought it could turn out to be a blessing. The horse returned after a few months along with another horse, and though it seemed like good luck, he thought it could eventually lead to misfortune. His son then broke his leg riding the new horse, which seemed like a setback, but a year later, there was a military draft and his son did not have to join in the fighting. The story shows that Saiweng's attitude, which was one of moderation, is a good one: when something seems like good luck, a reversal of fortune could come at any time, and vice versa. Restraining one's reaction to events is wise, since fate can always quickly swing the other way.

\section{Discussion}

We discuss three items that warrant further investigation.

\section{Something about satisfaction (manyi)}

While respondents seem to prefer the second highest response, there may be something different about satisfaction (manyi) in that respondents seem more likely to choose the 
highest level response, at least before 2007, when more than 20\% selected the highest level), which we do not see in the ladder or happiness (xingfu) variables (Table 3). Over time, however, satisfaction (manyi) seemed to have declined in China. Satisfaction (manyi) may be an easier question to answer than happiness (xingfu) because of its history, and it also may be easier to answer than the questions about emotions (happy kuaile and yukuai). Cross-cultural psychology shows that Chinese respondents think less frequently than Americans about whether their lives are happy, satisfying, or joyful (Diener et al. 1995).

\section{Comment on Easterlin et al. 2017}

Using different datasets and concepts (if each concept is analyzed as an individual trend line), we find support for Easterlin et al.'s (2017) argument that SWB in Chinese since the 1990 has basically taken on this U-shaped pattern, dipping in particular in the 2000-05 period and then rising again since.

However, we have a critique of Fig. 3.1 in their paper, where they present "Gallup1" before 2006 (based on the life satisfaction measure, manyi), and then continue the trend line with CGSS data on happiness (xingfu). We find that happiness (xingfu) and satisfaction (manyi) are only weakly correlated in WVS 1995, suggesting that the concepts in the survey could be different. Thus, combining the trend line may be problematic.

Easterlin relies on life satisfaction (manyi), happiness (xingfu), and a "ladder of life" question. Our data do not allow for comparisons between the "ladder" and the other terms used for SWB, but based on a qualitative understanding of "ladder" as pertaining to individual accomplishment more than social relationships going well, we think satisfaction (manyi) could be similar to the $\mathrm{BH}$ measure of satisfied (manyi) with job. Because satisfied (manyi) with job is only weakly correlated with the overall evaluation measures (happiness xingfu, good life hao shenghuo), positive emotion (happy kuaile) and meaning (yiyi), we caution against treating the "ladder" as the same as the others variables.

\section{Comment on "ladder of life" question in China}

Because Pew, which does the only publicly-available survey in China using the "ladder of life" question, only provides an urban sample, it is not comparable to the nationally-representative surveys we examine. However, since it is often used, sometimes in combination with other surveys, we offer some comments on what the question might evoke in respondents. The "ladder of life" survey question used by Pew asks an evaluative question. It asks the respondent to assess his or her well-being using a particular metaphor of an individual standing on a ladder. The wording of the question is:

Please imagine a ladder with steps numbered from 0 at the bottom to 10 at the top. Suppose we say that the top of the ladder represents the best possible life for you, and the bottom of the ladder represents the worst possible life for you. On which step of the ladder would you say you personally stand at this time?

This approach corresponds with Cantril's (1965) "Self-Anchoring Striving Scale," in which the good or bad life is hoped to be self-defined. The "ladder of life" question 
might correspond most to "engagement" and "accomplishment" in Seligman's concept of flourishing, but we could not compare as did not have any data sets where it overlapped with another SWB measure.

We do note, however, that in the symbolic continuum of the "ladder" question, going up is equated with as "best" and down as "worst," but what exactly the anchoring point at the top means is supposed to be up to the individual to define for himself. The ladder as a metaphor presents a metaphor for well-being that is conceptualized around an individual; there is no room, for example, for a family on a ladder. Because the "ladder" question does not encompass one's relationships, it may be a measure that is specific to an evaluation of the individual's own achievements or accomplishments.

\section{Conclusions}

This is a first effort to compare SWB measures, and we acknowledge that we are using simple methods to address a complex question. To answer our original questions of (1) whether we can use the different measures for subjective well-being interchangeably (2) whether they may work together as a construct, we draw upon our findings with regard to correlations between the terms and coefficients of reliability. The correlations between the SWB terms are relatively weak (under 0.53), showing that the measures are not interchangeable. That the modal response is the second highest category may indicate a tendency towards moderate responses. Since the Cronbach's alpha coefficients (at least in the BH data set) show that happiness (xingfu), happy (kuaile), good life (haoshenghuo), satisfied (manyi) with job, and meaning (yiyi) have shared covariance, they may therefore tap into a larger underlying concept. Our findings suggest that the different measures are not interchangeable, but they may be used together as a measure of human flourishing, consisting of different components, including positive emotion, social relationships going well, and meaning.

\section{Acknowledgments}

The authors wish to thank Chih-Jou Jay Chen, Deborah Davis, James Farrer, Richard Madsen, and Anna Sun for helpful comments, and Dana Drecksel for research assistance. We also thank the anonymous reviewers for their careful reading of our manuscript and their many insightful comments and suggestions. An earlier version of this paper was presented at the Association of Asian Studies 2014 annual meeting. This publication was made possible through the support of a grant from the John Templeton Foundation. The opinions expressed in this publication are those of the authors and do not necessarily reflect the views of the John Templeton Foundation.

\section{Authors' contributions}

$\mathrm{BH}$ designed the framework of the study and drafted the manuscript. WZ contributed to the theoretical framework and collected and coded the dataset. CK did the quantitative analysis: the interpretation of basic statistics in Table 3, the correlations in Table 4, and calculating the Cronbach's alpha coefficient for the five BH variables that we describe in the findings. All authors read and approved the final manuscript.

Competing interests

We confirm that this manuscript has not been published elsewhere and is not under consideration by another journal. The authors declare that they have no competing interests.

\section{Publisher's Note}

Springer Nature remains neutral with regard to jurisdictional claims in published maps and institutional affiliations.

Author details

${ }^{1}$ Sociology Department, Georgetown University, 3520 Prospect Street, NW, Suite 209, Washington DC 20057, USA.

${ }^{2}$ Sociology Department, St. Lawrence University, 23 Romoda Drive, Canton, NY 13617, USA. ${ }^{3}$ Department of

Government, Georgetown University, 37th and O Streets, N.W, Washington DC 20057, USA. 
Received: 5 October 2016 Accepted: 16 August 2017

Published online: 25 August 2017

\section{References}

Cantril, H. 1965 The pattern of human concerns. New Brunswick: Rutgers University Press

Chen, L. 2014. An expanding repertoire: The usages and meanings of the term fu and xingfu in pre-modern China. Philadelphia: Paper presented at the Association for Asian Studies Annual Conference.

Deaton, A. 2008. Income, health, and well-being around the world: Evidence from the Gallup world poll. Journal of Economic Perspectives. 22(2): 53-72.

Diener, E, E Suh, H Smith, S Liang. 1995. National differences in reported subjective well-being: Why do they occur? Social Indicators Research 34(1): 7-32.

Easterlin, R. 1974. Does economic growth improve the human lot? Some empirical evidence. Nations and households in economic growth 89: 89-125.

Easterlin, RA, LA McVey, M Switek, O Sawangfa, JS Zweig. 2010. The happiness-income paradox revisited. Proceedings of the National Academy of Sciences 107(52): 1-6.

Easterlin, RA, R Morgan, M Switek, F Wang. 2012. China's life satisfaction, 1990-2010. Proceedings of the National Academy of Sciences

Easterlin, Richard, Fei Wang, and Shun Wang. 2017. Growth and happiness in China, 1990-2015. World happiness report, ed. John Helliwell, Richard Layard, Jeffrey Sachs, 48-82.

Hagerty, MR, R Veenhoven. 2003. Wealth and happiness revisited - growing national income does go with greater happiness. Social Indicators Research 64(1): 1-27.

Krueger, A, D Schkade. 2008. The reliability of subjective well-being measures. Journal of Public Economics 92(8-9): 1833-1845.

Kwan, V, M Bond, T Singelis. 1997. Pancultural explanations for life satisfaction: Adding relationship harmony to self-esteem. Journal of Personality and Social Psychology 73: 1038-1051.

Seligman, M. 2011. Flourish: A visionary new understanding of happiness and well-being. New York: Simon and Schuster.

Suh, Eunkook. 2000. Culture and subjective well-being. In Culture and subjective well-being, ed. Ed Diener and Eunkook Suh, 63-86. Boston: MIT Press.

Suh, E, E Diener, S Oishi, H Triandis. 1998. The shifting basis of life satisfaction judgments across cultures: Emotions versus norms. Journal of Personality and Social Psychology 74(2): 482-493.

Sun, A. 2014 The official discourse of happiness in contemporary China. Philadelphia: Paper presented at the Association for Asian Studies Annual Conference.

$X_{i}$, J. 2013 About the China dream of realizing the renaissance of the Chinese nation. Beijing: The Central Party Documents Press.

\section{Submit your manuscript to a SpringerOpen ${ }^{\circ}$ journal and benefit from:}

- Convenient online submission

- Rigorous peer review

- Open access: articles freely available online

- High visibility within the field

- Retaining the copyright to your article 